\title{
Versatile one-step introduction of multiple hydrogen-bonding sites onto extended $\pi$-conjugated systems $\dagger$
}

\author{
Fu-Chuan Fang, ${ }^{a}$ Cheng-Che Chu, ${ }^{b}$ Chih-Hao Huang, ${ }^{b}$ Guillaume Raffy, ${ }^{b}$ André Del Guerzo, ${ }^{b}$ \\ Ken-Tsung Wong $*^{a}$ and Dario M. Bassani* ${ }^{b}$
}

Received (in Cambridge, UK) 6th August 2008, Accepted 30th September 2008

First published as an Advance Article on the web 6th November 2008

DOI: $10.1039 / b 813704 h$

\begin{abstract}
Unprotected arylbiuret units were smoothly introduced onto $\pi$-conjugated structures to afford new supramolecular materials leading to interpenetrated networks.
\end{abstract}

In the quest for new opto- and electro-active organic materials, the control of intermolecular interactions through designed supramolecular forces has become a major goal as it provides the chemist with a handle on controlling one of the principal parameters influencing the overall properties of the final material: its morphology. Thus, structural motifs coding for metal ion coordination, electrostatic forces, van der Waals interactions, or hydrogen-bonds have been coupled to a diversity of electroactive structures to afford compounds whose self-assembly is driven by the molecular recognition elements guided by solvent-solute interactions. ${ }^{1}$

For applications in the emergent field of molecular electronics, hydrogen-bonds (H-B) are particularly appealing because they are directional and do not interfere with the spectroscopic or electronic properties of the material. However, the introduction of $\mathrm{H}$-B elements onto compounds possessing extended $\pi$-conjugated structures can be challenging in cases where the presence of an inert or flexible alkyl spacer is not desirable. Direct connection of the H-B motif, such as in the case of a tetra H-B unit developed by Meijer and co-workers ${ }^{2}$ or in perylene diimides, ${ }^{3}$ benefits from the structural rigidity imparted by the conjugated unit and results in well-defined supramolecular architectures useful in promoting or maintaining electronic interactions. ${ }^{4}$ Ureas (and related bis-ureas, or biurets) are a class of selfcomplementary H-B elements coding for the formation of extended planar sheets that have been successfully used in the design of organogelators. ${ }^{5}$ However, the incorporation of a (thio)urea or biuret unit is contingent upon the availability of the corresponding (thio)isocyanate or amine precursors, which are not readily available or are prone to facile oxidation when directly connected to electron-rich aromatic platforms. ${ }^{6}$

We now report the use of Suzuki coupling methodology to smoothly introduce an aryl biuret H-B motif onto electron-

${ }^{a}$ Department of Chemistry, National Taiwan University, No 1, Sec. 4 Roosevelt Rd., 106 Taipei, Taiwan.E-mail:kenwong@ntu.edu.tw; Fax: 88623366 1667: Tel: 886233661665

${ }^{b}$ Institut des Sciences Moléculaires, CNRS UMR 5255, 33405

Talence, France.E-mail: d.bassani@ism.u-bordeaux1.fr,

Fax: 3354000 6158; Tel: 33540002827

+ Electronic supplementary information (ESI) available: Details of the synthesis and characterization $\left({ }^{1} \mathrm{H}\right.$ and ${ }^{13} \mathrm{C}$ NMR, IR, HRMS) of compounds 1-4, table of absorption and emission data of $\mathbf{2 - 4}$, confocal fluorescence images of neat films of $\mathbf{2 b}$ and $\mathbf{2 c}$. See DOI: $10.1039 / \mathrm{b} 813704 \mathrm{~h}$ rich conjugated oligomers. The strategy employed rests on the preparation of a new boronic acid derivative incorporating the desired unprotected biuret unit, thus avoiding both the necessity of isolating unstable arylamine derivatives and cumbersome protection/deprotection sequences. The reaction was successfully tested on terthiophene, ter(phenylenevinylene) and tetraphenylene thanks to the ready availability of the corresponding brominated oligomers. Additionally, Förster energy transfer was used to probe the effect of molecular recognition on the microheterogeneity of the thin films formed by the samples, revealing important variations induced by the biuret motifs.

Compound 1 (Scheme 1) was conceived to exploit the wellknown Suzuki-Miyaura coupling methodology as a means to introduce biuret motifs onto electron-rich conjugated oligomers without the use of inert/flexible alkyl spacers. Suzuki coupling is tolerant of primary and secondary amides, and is particularly adapted to oligothiophenes and oligophenylenes. ${ }^{7}$ As shown in Scheme 1, compound 1 was prepared in two steps from 4-bromoaniline. Following reaction with nitro-biuret to form the biuret $\mathrm{H}-\mathrm{B}$ motif, the aryl bromide thus obtained was converted to the boronic ester intermediate using bis(pinnacolato)diborane via $\mathrm{Pd}$-mediated borylation. ${ }^{8}$ This procedure does not require the generation of the corresponding lithiated species typically used to form arylboronates, which are incompatible with the presence of the unprotected biuret unit. The key intermediate, compound 1, was thus obtained in $60 \%$ isolated yield after recrystallization from ether/hexanes. $\ddagger$

To test the ability of $\mathbf{1}$ to undergo Suzuki coupling reactions with electron-rich conjugated oligomers, the palladiummediated cross-coupling of $\mathbf{1}$ with $\alpha, \omega$-dibromobisfluorene (2a), $\alpha, \omega$-dibromotertra(thiophene) (3a), and $\alpha, \omega$-dibromobis(styryl)benzene (4a) was tested under standard reaction conditions (Scheme 2). Compounds 2a, 3a, and 4a were selected as being representative of well-known oligofluorenes, oligothiophenes, and oligo(phenylene-vinylene)s in light of their importance in molecular electronics. Alkyl substituents
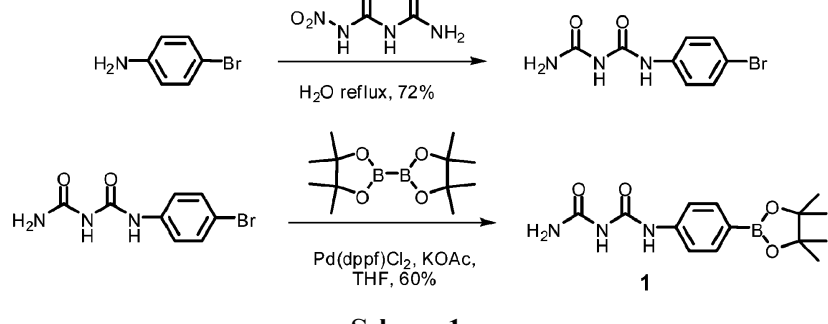

Scheme 1 


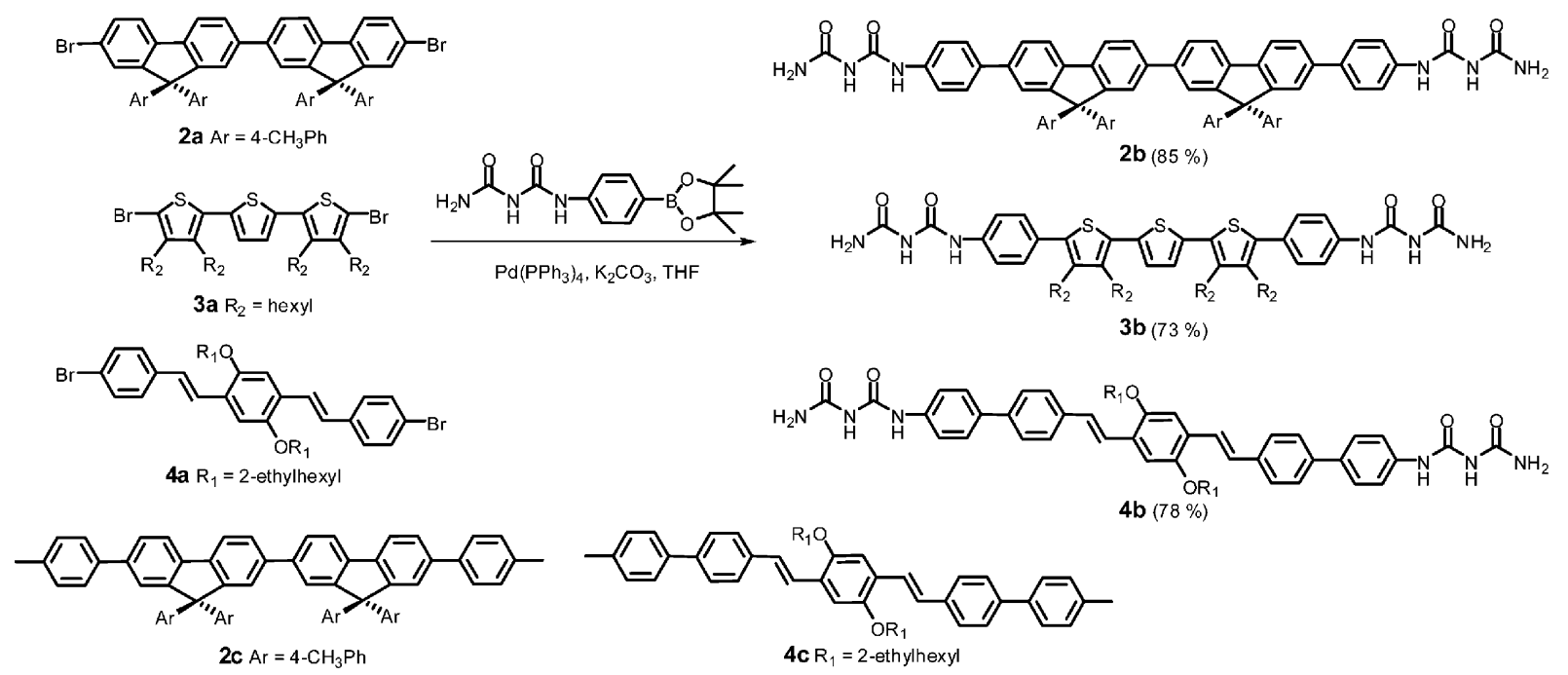

Scheme 2 Synthesis of compounds $\mathbf{2 b}, \mathbf{3 b}$ and $\mathbf{4 b}$, and the structures of model compounds $\mathbf{2 c}$ and $\mathbf{4} \mathbf{c}$.

were incorporated from the onset to ensure adequate solubility even after introduction of the H-B units. In all cases, the corresponding bis-substituted products $\mathbf{2 b}, \mathbf{3 b}$ and $\mathbf{4 b}$ were obtained in good yield, ranging from 73 to $85 \%$ (unoptimized isolated yields, see $\mathrm{ESI} \dagger$ ), which corresponds to $85-90 \%$ conversion per halogen atom.

The absorption and emission spectra of $\mathbf{2 b}$ and $\mathbf{4 b}$ are shown in Fig. 1, from which it can be deduced that the strong overlap between the emission of $\mathbf{2 b}$ and the absorption of $\mathbf{4 b}$ should be conducive to Förster resonant energy transfer (FRET) from electronically excited $\mathbf{2 b}$ (the donor) to $\mathbf{4 b}$ (the acceptor). FRET imaging has been extensively used in biology to discern spatial proximity between distal sites on proteins, or to observe binding or vectorization events, and has also been used to directly visualize spatial separation and phase boundaries between emissive donor and acceptor materials in bulk samples displaying heterogeneity. ${ }^{9}$ To ascertain the influence of the H-B motifs, reference compounds in which the biuret units were replaced by methyl substituents were also prepared (compounds $\mathbf{2 c}$ and $\mathbf{4 c}$, Scheme 2). Their absorption and emission spectra closely match those of $\mathbf{2 b}$ and $\mathbf{4 b}$ (see ESI $\dagger$ ).

Thin films of fluorene donor $\mathbf{2 b}$ or $\mathbf{2} \mathbf{c}$ containing a small amount of acceptor $\mathbf{4 b}$ or $\mathbf{4 c}(10 \mathrm{~mol} \%)$ were prepared by spincoating the appropriate mixtures from THF solutions on glass substrates. Upon deposition, the absorption and emission

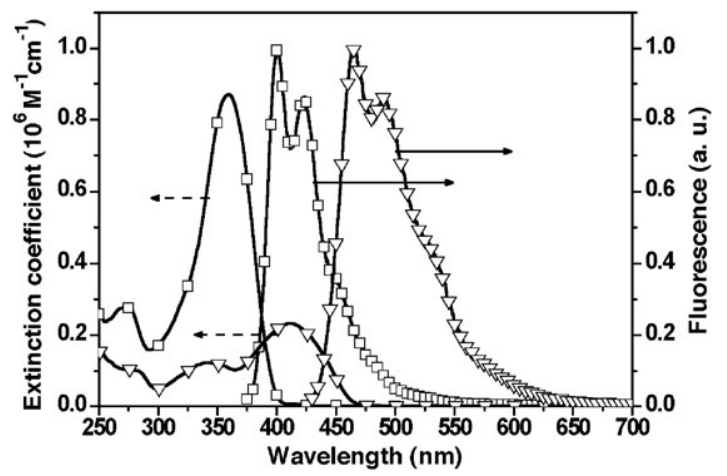

Fig. 1 Absorption and fluorescence spectra of $\mathbf{2 b}$ (squares) and $\mathbf{4 b}$ (triangles) in THF $\left(1.0 \times 10^{-5} \mathrm{M}\right)$. spectra of $\mathbf{2 b}$ and $\mathbf{4 b}$ are only slightly shifted with respect to solution (see ESI $\dagger$ ). Although all the films thus obtained were homogeneous in appearance, confocal fluorescence microscopy images of the films reveals strong variations in the microheterogeneity of the samples (Fig. 2). This is readily seen by selectively exciting the donor and collecting separately donor and acceptor emission. In samples $\mathrm{A}(\mathbf{2 c} / \mathbf{4 c})$ and $\mathrm{B}$ $(\mathbf{2 c} / \mathbf{4 b})$, where the donor $\mathbf{2 c}$ does not possess biuret motifs, similar intensity distributions are found for the residual emission of the donor and the main emission resulting from the acceptor. In both cases, the intensity is the highest in polydisperse globular aggregates whose size ranges from $c a$. $280 \mathrm{~nm}$ (lower limit of instrumental resolution) to microns in diameter.§ In sample B, intense emission from homogeneously dispersed biuret-acceptor $\mathbf{4 b}$ is also seen from the remaining area not occupied by the large aggregates. A globally uniform film (within instrumental resolution) appears to be formed with biuret-containing donor $\mathbf{2 b}$ when biuret-free acceptor $\mathbf{4 c}$ is incorporated (sample $\mathrm{C}, \mathbf{2 b} / \mathbf{4 c}$ ). This suggests that donor-acceptor mixtures in which the major component contains the biuret $\mathrm{H}-\mathrm{B}$ element provide an environment that is more tolerant of the presence of dispersed acceptor. In sharp contrast, the presence of biuret motifs on both the donor and the acceptor $\mathbf{4 b}$ (sample D, $\mathbf{2 b} / \mathbf{4 b}$ ) induces the formation of a highly interpenetrated network of the two materials characterized by areas of acceptor emission displaying widths ranging from $\leq 260 \mathrm{~nm}$ to $c a .400 \mathrm{~nm}$.

To further explore the dispersion of $\mathbf{4 b}$ into $\mathbf{2 b}$, films in which the loading ratio is varied from $1 \%$ to $10 \%(\mathbf{4 b}: \mathbf{2 b}$,

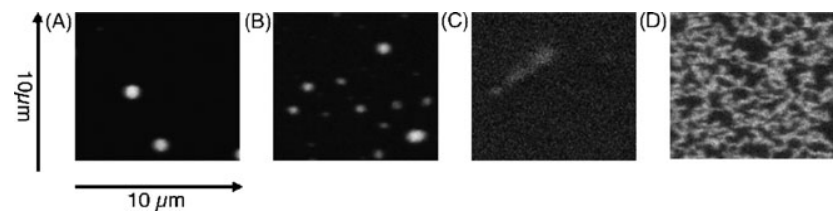

Fig. 2 Fluorescence confocal microscopy images of films of mixed donors (2b and $\mathbf{2 c}$ ) and acceptors (4b and $\mathbf{4 c}$ ); sample A: $\mathbf{2 c} / \mathbf{4 c}$; B: $\mathbf{2 c} / \mathbf{4 b}$; C: $\mathbf{2 b} / \mathbf{4 c} ;$ D: $\mathbf{2 b} / \mathbf{4 b}(10 \mathrm{~mol} \%$ acceptor doping in donor); $\lambda_{\mathrm{ex}}=385 \mathrm{~nm}, \lambda_{\mathrm{obs}}=470-550 \mathrm{~nm}$. 


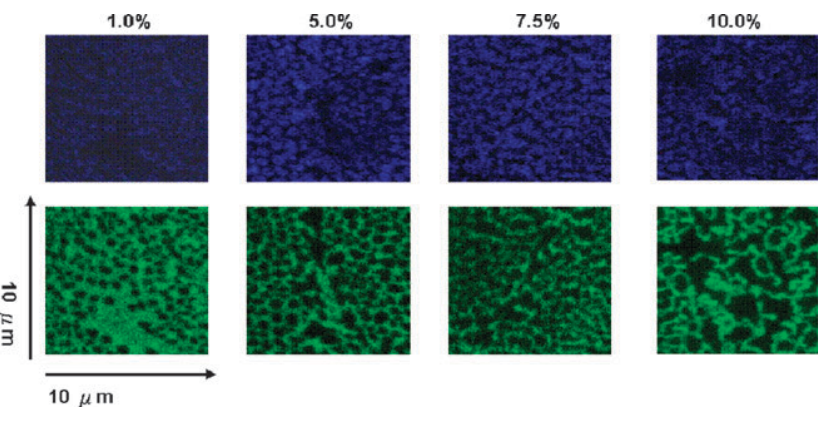

Fig. 3 Confocal fluorescence images of films of $\mathbf{2 b}$ incorporating varying amounts (1-10\%) of $\mathbf{4 b}$ obtained by selectively exciting $\mathbf{2 b}$ at $385 \mathrm{~nm}$. Residual emission from unquenched $\mathbf{2 b}$ is collected in the blue channel (top), whereas emission from $\mathbf{4 b}$ (via FRET) is collected in the green channel (bottom).

Fig. 3) were investigated. It is immediately obvious that the emission collected from the blue channel (400-450 nm), originating from the fluorene donor, and the green channel (470-550 nm), originating from FRET to the acceptor, are complementary and highly contrasted, indicating two well separated phases. In one of these phases, the acceptor is almost absent and emission originating from unquenched donor is collected in the blue channel. In contrast, the second phase shows exclusively the emission from the acceptor. The overall surface area of this latter phase is significantly lager than the proportion of acceptor in the mixture, indicating that it must be a blend of donor and acceptor in which complete $\mathbf{2 b}$ to $\mathbf{4 b}$ FRET occurs. This phase covers $c a .85 \%$ of the film area at $1 \%$ doping, and decreases to $c a .50 \%$ as the proportion of acceptor is increased to $10 \%$ doping. This shows that selfaggregation of the acceptor is favored at higher concentrations of $\mathbf{4 b}$, and that the level of dispersion decreases accordingly.

In general, compared to larger, globular aggregates in which surface area is minimized, interpenetrated networks increase the contact area between two materials and are less favorable energetically. Such networks are of interest for applications where large areas of contact between two materials is desirable, such as in photovoltaic devices, where exciton dissociation occurs at the electron donor-acceptor interface. The propensity of $\mathbf{2 b}$ and $\mathbf{4 b}$ to readily form segregated networks $v s$. globular aggregates originates from segregation of the components during the evaporation process, and is presumably a consequence of the relative strengths of the intermolecular forces between identical vs. mixed subcomponents. The presence of the biuret units may be expected to promote the dispersion of the acceptor into the donor material due to the strong H-B interactions they instill, overriding weaker $\pi$-staking interactions between the aromatic residues in $\mathbf{2} \mathbf{b}$ and 4b. The occurrence of strong aggregation is supported by the significant broadening of the proton signals in the ${ }^{1} \mathrm{H}$ NMR spectrum of $\mathbf{2 b}$ and $\mathbf{4 b}$ (but not $\mathbf{2 c}$ and $\mathbf{4 c}$ ) in $\mathrm{CDCl}_{3}$ vs. DMSO-d 6 .
In conclusion, we have devised a general strategy for the efficient introduction of biuret $\mathrm{H}$-B units onto $\pi$-conjugated platforms for which the corresponding primary amines are not available. In the case of the bis-fluorene/bis(styryl)phenylene system, the biuret units impart molecular recognition properties that result in the formation of interdispersed network phases in which energy transfer is highly efficient.

We thank the NSC (Taiwan) and MESR (France) for support (PHC Orchid). Funding from the CNRS (to D. M. B.) is gratefully acknowledged.

\section{Notes and references}

$\ddagger$ A mixture of 4-bromobenzenebiuret (1.03 g, $4 \mathrm{mmol}$ ), bis(pinacolato)diboron $(1.52 \mathrm{~g}, 6 \mathrm{mmol})$, KOAc $(1.16 \mathrm{~g}, 11.8 \mathrm{mmol}), \mathrm{Pd}(\mathrm{dppf}) \mathrm{Cl}_{2}$ $(0.15 \mathrm{~g}, 0.2 \mathrm{mmol})$ in THF $(30 \mathrm{~mL})$ was refluxed for two days. The reaction was quenched with water and extracted with THF. Evaporation of the solvent and recrystallization from ether-hexane afforded 1 as a pale white solid $(0.7 \mathrm{~g}, 60 \%)$.

$\S$ A similar behaviour is also observed in films of neat $\mathbf{2 c}$.

1 (a) For recent reviews of this topic, see: F. J. M. Hoeben, P. Jonkheijm, E. W. Meijer and A. P. H. J. Schenning, Chem. Rev., 2005, 105, 1491; (b) A. Ajayaghosh and V. K. Praveen, Acc. Chem. Res., 2007, 40, 644; (c) S. Yagai, J. Photochem. Photobiol., C, 2006, 7, 164; (d) C. H. Huang and D. M. Bassani, Eur. J. Org. Chem., 2005, 4041.

2 A. P. H. J. Schenning, P. Jonkheijm, E. Peeters and E. W. Meijer, J. Am. Chem. Soc., 2001, 123, 409.

3 (a) T. E. Kaiser, H. Wang, V. Stepanenko and F. Würthner, Angew. Chem., Int. Ed., 2007, 46, 5541; (b) S. Yagai, T. Seki, T. Karatsu, A. Kitamura and F. Wurthner, Angew. Chem., Int. Ed., 2008, 47, 3367.

4 (a) N. D. McClenaghan, Z. Grote, K. Darriet, M. Zimine, R. M. Williams, L. De Cola and D. M. Bassani, Org. Lett., 2005, 7, 807; (b) C. H. Huang, N. D. McClenaghan, A. Kuhn, J. W. Hofstraat and D. M. Bassani, Org. Lett., 2005, 7, 3409.

5 (a) S. Boileau, L. Bouteiller, F. Laupretre and F. Lortie, New J. Chem., 2000, 24, 845; (b) O. Colombani and L. Bouteiller, New J. Chem., 2004, 28, 1373; (c) E. Obert, M. Bellot, L. Bouteiller, F. Andrioletti, C. Lehen-Ferrenbach and F. Boue, J. Am. Chem. Soc., 2007, 129, 15601; (d) F. Vonau, D. Suhr, D. Aubel, L. Bouteiller, G. Reiter and L. Simon, Phys. Rev. Lett., 2005, 94, 4; (e) O. J. Dautel, M. Robitzer, J. P. Lere-Porte, F. Serein-Spirau and J. J. E. Moreau, J. Am. Chem. Soc., 2006, 128, 16213; (f) X. Don, W. Pisula, J. Wu, G. J. Bodwell and K. Mullen, Chem. Eur J., 2008, 14, 240.

6 For a recent example of a urea-phenylene-vinylene derivative prepared via a primary amine, see: S. Yagai, S. Kubota, T. Iwashima, K. Kishikawa, T. Nakanishi, T. Karatsu and A. Kitamura, Chem. Eur. J., 2008, 14, 5246.

7 (a) H. Doucet, Eur. J. Org. Chem., 2008, 2013; (b) C. A. Fleckenstein and H. Plenio, Chem. Eur. J., 2008, 14, 4267.

8 (a) T. Ishiyama and N. Miyaura, J. Organomet. Chem., 2000, 611, 392; (b) T. Ishiyama, M. Yamamoto and N. Miyaura, Chem. Commun., 1997, 689.

9 (a) F. J. M. Hoeben, A. P. H. J. Schenning and E. W. Meijer, ChemPhysChem, 2005, 6, 2337; (b) F. J. M. Hoeben, I. O. Shklyarevskiy, M. J. Pouderoijen, H. Engelkamp, A. P. H. J. Schenning, P. C. M. Christianen, J. C. Maan and E. W. Meijer, Angew. Chem., Int. Ed., 2006, 45, 1232; (c) A. Del Guerzo, A. G. L. Olive, J. Reichwagen, H. Hopf and J.-P. Desvergne, J. Am. Chem. Soc., 2005, 127, 17984. 